\title{
Eficiência de sistemas alagados construídos na remoção de poluentes de águas residuárias da suinocultura
}

(doi:10.4136/ambi-agua.142)

\author{
Antonio Teixeira de Matos ${ }^{1}$; Wallison da Silva Freitas²; Paola Alfonsa Vieira Lo \\ Monaco $^{3}$ \\ ${ }^{1}$ Universidade Federal de Viçosa - UFV \\ E-mail: atmatos@ufv.br \\ ${ }^{2}$ Instituto Federal de Educação, Ciência e Tecnologia do Norte de Minas Gerais - IFNMG \\ E-mail: wallfreitas@yahoo.com.br \\ ${ }^{3}$ Instituto Federal de Educação, Ciência e Tecnologia do Espírito Santo - IFES \\ E-mail: paolalomonaco2004@yahoo.com.br
}

\section{RESUMO}

Objetivou-se, com a realização deste trabalho, avaliar a eficiência de sistemas alagados construídos (SAC(s)) na remoção de poluentes em sistemas com monocultivo e multicultivo não consorciado, com três diferentes espécies de plantas, no tratamento de água residuária da suinocultura (ARS). Para isso, foram construídos 5 SAC(s) de 24,0 m x 1,1 m x 0,7 m, impermeabilizados com geomembrana de policloreto de vinila (PVC) e preenchidos com 0,4 $\mathrm{m}$ de brita zero. Nos SAC1, SAC2 e SAC3 cultivou-se a taboa (Typha latifolia L.), a alternantera (Alternanthera philoxeroides (Mart.) Griseb.) e o capim tifton-85 (Cynodon dactylon Pers.), respectivamente. No SAC4, foi plantada a Alternanthera no $1^{\circ}$ terço, a taboa no $2^{\circ}$ terço e o capim tifton-85 no $3^{\circ}$ terço do leito. O SAC5 não foi cultivado e serviu como testemunha sem plantas. Após passar por um filtro orgânico preenchido com bagaço de canade-açúcar picado, a ARS foi aplicada nos SAC(s) numa vazão de $0,8 \mathrm{~m}^{3} \mathrm{~d}^{-1}$, o que correspondeu a um tempo de detenção hidráulica de 4,8 dias. De acordo com os resultados obtidos, pôde-se concluir que os cinco SAC(s) tiveram eficiências de remoção de poluentes estatisticamente iguais entre si, tais sistemas foram mais eficientes na remoção de SST, DQO, $\mathrm{DBO}_{\text {total }}$ e Zn, apresentando valores médios de 91, 89, 86 e 94\%, respectivamente. Remoções relativamente altas também foram obtidas de ST, $\mathrm{N}$-total, $\mathrm{NH}_{4}{ }^{+}$e P-total, tendo sido obtidos valores médios de 62, 59, 52 e 50\%, respectivamente. As plantas se equivaleram entre si, em termos de conferir eficiência ao sistema e o SAC não cultivado apresentou capacidade semelhante aos SAC(s) cultivados na remoção de poluentes da ARS.

Palavras-chave: tratamento de resíduos; dejeto de suínos; sistemas wetlands; leitos cultivados.

\section{Performance of the constructed wetland systems in pollutants removal from hog wastewater}

\section{ABSTRACT}

The main objective of this work was to evaluate the efficiency of a constructed wetland systems (CWS) for pollutants removal, in mono crop and multi crop with three different species of plants, originated from hog wastewater treatment (HW). Therefore, 5 CWS of $24.0 \mathrm{~m} \times 1.1 \mathrm{~m} \times 0.7 \mathrm{~m}$ were constructed, sealed with a membrane of polyvinyl chloride (PVC) and filled with $0.4 \mathrm{~m}$ of small gravel. In CWS1, CWS2 and CWS3 grown to cattail (Typha latifolia L.), Alternanthera philoxeroides (Mart.) Griseb. and Tifton 85 grass (Cynodon dactylon Pers.), respectively. In the bed of CWS4 was planted at $1^{\text {st }}$ third Alternanthera, cattail, in the $2^{\text {nd }}$ third and tifton- 85 grass and in the $3^{\text {rd }}$ third of. The CWS5 
was not planted and it was used as control. After passing through a filter filled with crushed bagasse of sugar cane, the HW was applied to the CWS in a flow of $0.8 \mathrm{~m}^{3} \mathrm{~d}^{-1}$, which corresponded to a hydraulic detention time of 4.8 days. According to the results it was shown that the five CWS(s) had statistically nearly the same removal of pollutants, and the average removal efficiency of TSS, COD, BOD and Zn, were 91, 89, 86 and 94\%, respectively. Also high removals were obtained concerning the ST, $\mathrm{N}$-total, $\mathrm{NH}_{4}{ }^{+}$and P-total, with average values of 62, 59, 52 and 50\%, respectively. The plants in all planted CWS worked in a similar way maintaining the system efficiency and the non cultivated CWS presented analogous capacity of pollutants removal when compared to the cultivated CWS(s).

Keywords: waste management; pig waste; wetlands systems; cultivated beds.

\section{INTRODUÇÃO}

A suinocultura é, no Brasil, uma atividade socioeconômica importante, por gerar grande quantidade de empregos diretos e indiretos e por produzir alimentos altamente protéicos e de boa qualidade para o consumo humano. Nos últimos anos, a atividade da suinocultura se expandiu intensamente, o que pode ser atribuído ao pequeno espaço que os suínos necessitam para seu crescimento e desenvolvimento e por constituírem, após o abate, fonte de proteína barata e saborosa (Tobias, 2002). No entanto, o desenvolvimento da suinocultura tem apresentado, como maior empecilho, o alto potencial poluidor da atividade, decorrente da produção concentrada de dejetos dos animais.

Além da poluição das águas superficiais e subterrâneas, outros riscos potenciais para o ambiente, pela aplicação de doses inadequadas de resíduos orgânicos, são a salinização e a poluição do solo e a contaminação de plantas com metais pesados e outros compostos orgânicos, efeitos prejudiciais à estrutura e macroporosidade do solo e a contaminação de homens e animais com agentes patogênicos (Matos e Sediyama, 1995).

Dentre as diversas soluções propostas para tratamento de águas residuárias ricas em material orgânico, distingue-se a sua disposição em SAC(s), por ser uma forma viável e barata para seu tratamento.

Dentre os componentes fundamentais dos SAC(s) estão as macrófitas aquáticas, o substrato e o biofilme de bactérias formado no meio, responsáveis, direta ou indiretamente, pela ocorrência dos mecanismos de remoção de poluentes associados a esses sistemas (Marques, 1999).

Estudos em escala real e experimental têm mostrado que os SAC(s) possuem grande capacidade na redução de poluentes. Em geral, essa redução é decorrente de mecanismos físicos, químicos e biológicos, incluindo-se processos de sedimentação, filtração, absorção, precipitação e adsorção química, interações microbianas, extração pelas plantas, volatilização e complexação (Marques, 1999; Wood, 1995).

Os principais mecanismos para remoção de SST e $\mathrm{DBO}_{\text {total }}$ são a floculação, a sedimentação e a filtração de sólidos suspensos e das partículas coloidais de maior tamanho. Os SAC(s) agem como filtro horizontal, de modo a favorecer a separação de SST por sedimentação (discreta e floculenta), ocorrendo o aprisionamento físico e adsorção por meio de biofilmes aderidos ao meio suporte e raízes desenvolvidas nesse meio. Os SAC(s) são eficientes em função da baixa velocidade de escoamento e da grande área específica do meio suporte (U.S. EPA, 2000). Resultados obtidos na República Tcheca evidenciaram eficiência de remoção de $81 \%$, $89 \%$ e $91 \%$ com respeito a DQO, $\mathrm{DBO}_{\text {total }}$ e SST, respectivamente em SAC utilizado no tratamento de esgoto doméstico (Vymazal, 2004). No Brasil, foram obtidas remoções de $48 \%$ a $77 \%$ e $40 \%$ a $81 \%$ na DQO e nos SST, respectivamente (Valentim, 2003), utilizando tempos de detenção hidráulica de 1 a 6 dias, e de $76 \%$ a 84\% na DQO, utilizando 3 a 6 horas de tempo de detenção (Sousa et al., 2000). Ainda no Brasil, Brasil et al. (2007), utilizando SAC(s) cultivados com taboa com tempo de detenção hidráulica de 1,9 e 3,8 dias, obtiveram, respectivamente, remoções de 86 e 90\% na DQO, 91\% nos SST (em ambos os 
tempos de detenção hidráulica) e de 80 e $86 \%$ na turbidez. Todos esses resultados foram obtidos no tratamento de esgoto doméstico.

Em SAC(s), a remoção do nitrogênio ocorre por meio da ação de micro-organismos, absorção e síntese vegetal. Nesses sistemas, a remoção de N-Total ocorre por meio da colheita da vegetação e por perdas para a atmosfera. Para Reed et al. (1995), a maior parte do nitrogênio removido ocorre por processos de nitrificação e desnitrificação, o que foi ratificado por Mander et al. (2004). A U.S. EPA (2000) considera baixa a remoção de nitrogênio via nitrificação e absorção pelas plantas, em comparação às cargas típicas aportadas em SAC(s), reportando taxas de remoção de 0,03 a $0,3 \mathrm{~g} \mathrm{~m}^{-2} \mathrm{~d}^{-1}$. Juwarkar (1995) obteve em SAC(s) de escoamento subsuperficial vertical, cultivados com Typha latifolia e Phragmites carca, remoções de N-total entre 65 e $73 \%$, enquanto que no SAC não cultivado tal remoção esteve entre 20 e 27\%. Maiores remoções de N-total e N-amônio em SAC(s) cultivados (Typha augustifolia) também foram verificados por Lim et al. (2001), com taxas de extração de 4,5 $\pm 0,6 \mathrm{~kg} \mathrm{ha}^{-1} \mathrm{dia}^{-1}$, correspondendo a $26 \pm 8 \%$ do total de nitrogênio aplicado. Segundo Lautenschlager (2001), a eficiência na remoção de nitrato total em SAC(s) está entre 1 e 34\%.

No SAC, a separação físico-química de fósforo pode iniciar com a deposição de sedimento, via sedimentação particulada, precipitação química e pela constrição de raízes das plantas (U.S. EPA, 2000). O fosfato solúvel pode ser adsorvido ao biofilme, que se desenvolve aderido ao meio suporte e às raízes de plantas, ou nos sedimentos (Sousa et al., 2001). As trocas de fosfato solúvel e adsorvido ocorrem por difusão e por sorção/dessorção, constituindo a principal forma de mobilidade do fosfato solúvel em SAC(s). Entretanto, os fosfatos podem ser precipitados como sais fosfato de ferro, de alumínio ou de cálcio, que são de baixa solubilidade, podendo ser adsorvido às partículas de argila, turfa ou óxidos e hidróxidos de ferro ou de alumínio, vindo a incorporar-se nos sedimentos (Reed et al., 1995; Arias e Brix, 2004). Juwarkar (1995) obteve remoções de 28 a 41\% na concentração de fósforo total em SAC(s) vegetados com Typha latifolia e Phragmites carca, enquanto que no SAC não vegetado a redução foi de apenas 12 a 17\%. Brasil et al. (2007) obtiveram remoções de 35\% e 48\% de P em SAC(s) cultivados com Typha, no tratamento de esgoto doméstico.

Nelson et al. (2002) obtiveram grande eficiência na remoção de $\mathrm{Cu}$ em SAC(s) cultivados com Scirpus californicus e tempo de detenção hidráulica de 2 dias. A remoção de Hg aumentou com o tempo de maturação dos SAC(s). Lim et al. (2001) obtiveram remoções menores que $0,5 \%$ em SAC(s) de escoamento superficial cultivados com taboa (Typha augustifolia). No tratamento com escoamento subsuperficial, tais remoções foram de 52-62\% e 59-67\%, respectivamente para o sistema cultivado e não cultivado. Shutes (2001) verificou, no primeiro ano de operação de um SAC, utilizado no tratamento de esgoto doméstico, na Inglaterra, eficiências de 10 a 99\%, 94 a 97\%, 89 a 97\% e 10 a 99\%, respectivamente, na remoção de $\mathrm{Cd}, \mathrm{Cu}, \mathrm{Pb}$ e $\mathrm{Zn}$.

Objetivou-se neste trabalho avaliar a eficiência de SAC(s) na remoção de poluentes oriundos de ARS em sistemas com monocultivo e multicultivo não consorciado, com três diferentes espécies de plantas.

\section{MATERIAL E MÉTODOS}

O experimento foi implantado na área experimental de hidráulica, irrigação e drenagem do Departamento de Engenharia Agrícola da Universidade Federal de Viçosa - DEA/UFV, em Viçosa, Minas Gerais.

A água residuária da suinocultura (ARS) usada no experimento foi captada, por bombeamento, de um tanque de alvenaria de dimensões de $(4 \times 5 \times 2)$ metros, utilizado para seu armazenamento, localizado próximo à referida área experimental. Antes de bombeá-la para a área experimental, fazia-se a mistura da ARS por meio de recirculação da ARS, a fim de homogeneizá-la no tanque. 
MATOS, A. T.; FREITAS, W. S.; LO MONACO, P. A. V. Eficiência de sistemas alagados construídos na remoção de poluentes de águas residuárias da suinocultura. Ambi-Agua, Taubaté, v. 5, n. 2, p. 119-132, 2010. (doi:10.4136/ambi-agua.142)

Avaliaram-se cinco SAC(s) de escoamento horizontal subsuperficial, constituídos por valas escavadas no solo, com dimensões de $0,7 \mathrm{~m}$ de altura (sendo 0,40 $\mathrm{m}$ de meio suporte), 1,1 $\mathrm{m}$ de largura e 24,0 m de comprimento, impermeabilizadas com geomembrana de PVC, com espessura de 0,5 mm. Como meio de suporte, utilizou-se brita 0, a qual apresentou volume de vazios de $48,4 \%$ e condutividade hidráulica do meio saturada (Ks) igual a $7.970 \mathrm{~m}$ $\mathrm{d}^{-1}$. Todos os SAC(s) tinham declividade do fundo de $0,005 \mathrm{~m} \mathrm{~m}^{-1}$ e o nível da água foi mantido rente ao meio suporte do final dos SAC(s).

A fim de proceder ao tratamento primário, a água residuária, antes de ser aplicada nos SAC(s), passou por filtro orgânico constituído por leito de bagaço de cana-de-açúcar picado, seguindo-se recomendações de Magalhães et al. (2006).

A distribuição dos tratamentos foi feita da seguinte forma: SAC1: totalmente cultivado com taboa (Typha latifolia L.); SAC2 cultivado com Alternanthera (Alternanthera philoxeroides (Mart Griseb); SAC3: cultivado com capim Tifton 85 (Cynodon dactylon (L.) Pers.); SAC4: cultivado no primeiro terço com Alternanthera, no segundo terço com taboa e no terço final com capim Tifton-85; e SAC5: sem cultivo. Na Figura 1 está apresentado o perfil esquemático da Estação Experimental de Tratamento.

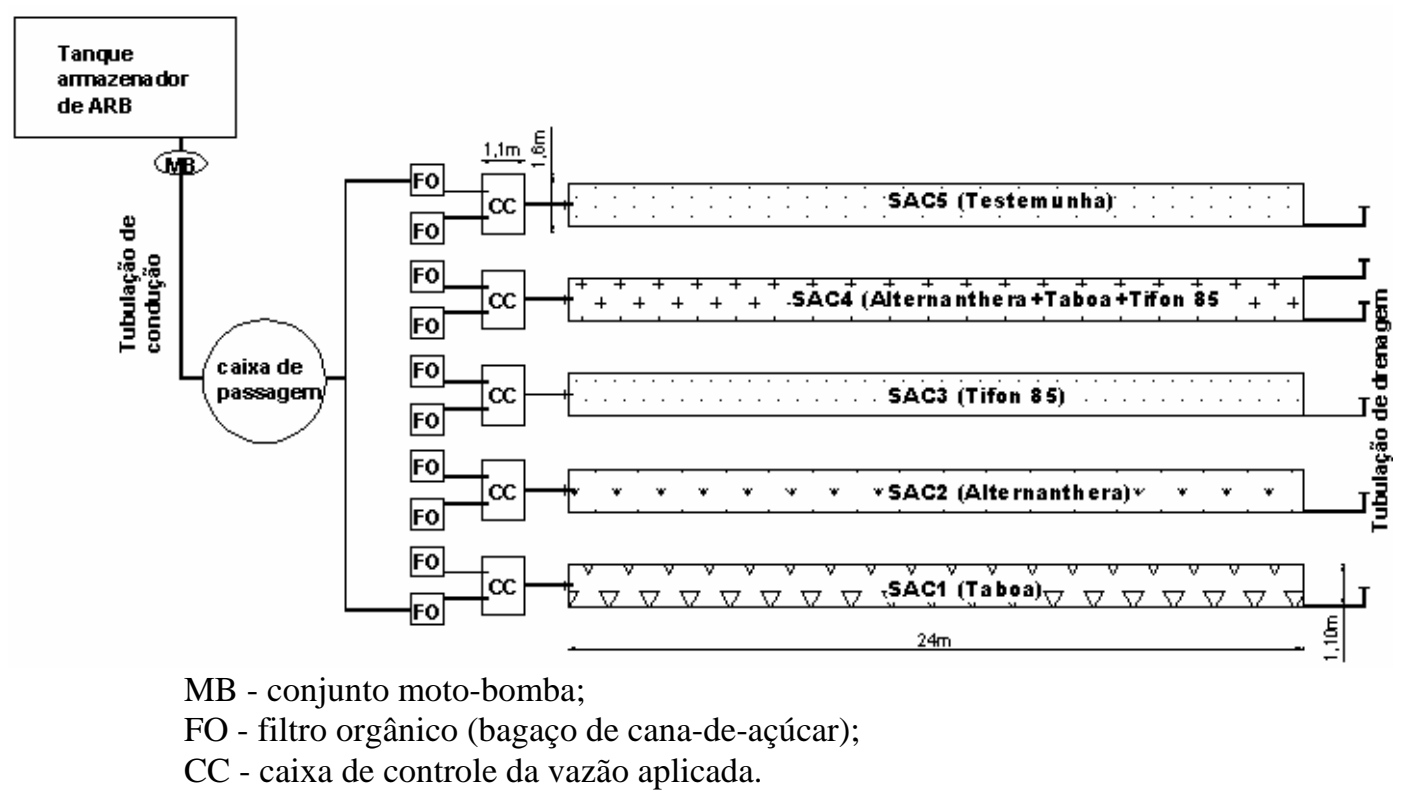

Figura 1. Perfil esquemático da Estação Experimental de Tratamento.

Em cada SAC foram aplicados $0,8 \mathrm{~m}^{3} \mathrm{~d}^{-1}$ de ARS filtrada, distribuindo-se a aplicação, em igual vazão, ao longo do dia, correspondendo a um tempo de detenção hidráulica aproximado de 4,8 dias. Baseado nas análises do efluente dos filtros orgânicos, feitas ao longo do período de experimentação, o volume aplicado nos SAC(s) correspondeu a uma taxa de aplicação média de $154,8 \mathrm{~kg} \mathrm{ha}^{-1} \mathrm{~d}^{-1}$ de $\mathrm{DBO}_{\text {total, }}$, conforme pode ser visto na Tabela 1. 
MATOS, A. T.; FREITAS, W. S.; LO MONACO, P. A. V. Eficiência de sistemas alagados construídos na remoção de poluentes de águas residuárias da suinocultura. Ambi-Agua, Taubaté, v. 5, n. 2, p. 119-132, 2010. (doi:10.4136/ambi-agua.142)

Tabela 1. Carga de nutrientes aplicada nas onze amostragens e média dessas amostragens $\left(\mathrm{kg} \mathrm{ha}^{-1} \mathrm{~d}^{-1}\right)^{*}$.

\begin{tabular}{|c|c|c|c|c|c|c|c|c|c|c|c|c|}
\hline \multirow{3}{*}{ Variável } & \multicolumn{12}{|c|}{ Carga de nutrientes $\left(\mathrm{kg} \mathrm{ha}^{-1} \mathrm{~d}^{-1}\right)^{* *}$} \\
\hline & $\mathbf{1}^{\mathrm{a}}$ & $2^{a}$ & $3^{a}$ & $4^{a}$ & $5^{a}$ & $6^{a}$ & $7^{a}$ & $8^{a}$ & $9^{a}$ & $10^{\mathrm{a}}$ & $11^{\mathrm{a}}$ & \multirow{2}{*}{ média } \\
\hline & $17 / 02$ & $14 / 03$ & 29/03 & $05 / 05$ & $18 / 05$ & 01/06 & $16 / 06$ & 04/07 & $18 / 07$ & $16 / 08$ & 01/09 & \\
\hline DQO & 233,2 & 561,5 & 649,6 & 135,2 & 808,1 & 1008,1 & 1331,1 & 350,6 & 544,6 & 473,3 & 404,4 & 590,9 \\
\hline $\mathrm{DBO}_{\text {total }}$ & - & 175,2 & 159,0 & 38,7 & 169,1 & 167,9 & 354,2 & 86,5 & 183,8 & 128,8 & 84,4 & 154,8 \\
\hline N-total & 72,7 & 50,1 & 89,5 & 65,2 & 113,7 & 124,4 & 113,9 & 101,5 & 109,0 & 85,4 & 101,1 & 93,3 \\
\hline Amônio & 95,2 & 21,4 & 12,6 & 66,2 & 76,7 & 76,0 & 85,2 & 87,9 & 65,5 & 55,9 & 94,7 & 67,0 \\
\hline Nitrato & 0,49 & 0,24 & 1,02 & 2,56 & 0,09 & 0,16 & 0,11 & 0,14 & 0,10 & 0,13 & 0,12 & 0,47 \\
\hline P-total & 10,5 & 13,4 & 24,2 & 15,2 & 26,3 & 33,8 & 28,9 & 21,4 & 26,7 & 21,4 & 21,7 & 22,1 \\
\hline K & 17,3 & 18,5 & 25,9 & 27,6 & 38,7 & 41,7 & 56,2 & 42,3 & 50,2 & 37,1 & 43,6 & 36,3 \\
\hline $\mathrm{Na}$ & 5,91 & 6,12 & 7,21 & 9,33 & 13,4 & 14,4 & 15,3 & 12,5 & 14,7 & 12,1 & 13,9 & 11,3 \\
\hline $\mathrm{Cu}$ & 0,04 & 0,16 & 0,28 & 0,10 & 0,16 & 0,56 & 0,22 & 0,03 & 0,35 & 0,50 & 0,32 & 0,25 \\
\hline $\mathrm{Zn}$ & 0,03 & 2,22 & 3,16 & 0,02 & 2,49 & 2,61 & 2,22 & 0,63 & 2,04 & 1,01 & 0,94 & 1,58 \\
\hline
\end{tabular}

*Valores baseados na média das amostras coletadas nos pontos efluentes ao filtro-orgânico (afluente aos

SAC(s)).

** Calculada com base no que foi obtido analisando-se 11 amostras de ARS.

As amostras de água foram coletadas antes dos SAC(s) (efluente dos filtros orgânicos), dentro de cada uma das cinco caixas de controle, e na saída do efluente de cada SAC.

Durante as coletas, que se realizaram em média a cada 15 dias, as amostras foram acondicionadas em caixas térmicas e, em seguida, conduzidas ao Laboratório de Qualidade da Água do DEA/UFV, para análise em conformidade com recomendações contidas no Standard Methods for the Examination of Water and Wastewater (APHA, 2005).

As variáveis avaliadas e os respectivos métodos estão apresentados na Tabela 2.

Tabela 2. Variáveis avaliadas e os respectivos métodos utilizados nas análises.

\begin{tabular}{l|l}
\hline Variáveis & Metodologia \\
\hline Condutividade elétrica (CE) & Condutivímetro de bancada \\
Demanda bioquímica de oxigênio & $\begin{array}{l}\text { Quantificação do oxigênio dissolvido pelo } \\
\text { método iodométrico (Processo Winkler) }\end{array}$ \\
$\left(\mathrm{DBO}_{\text {total }}\right)$ & Método de oxidação química em refluxo aberto \\
Demanda química de oxigênio (DQO) & Método gravimétrico \\
Sólidos totais (ST) & Método gravimétrico \\
Sólidos suspensos totais (SST) & Diferença entre os ST e os SST \\
Sólidos dissolvidos totais (SDT) & Processo semimicro Kjeldahl \\
Nitrogênio total (N $\left.\mathrm{N}_{\text {Total }}\right)$ & Uso do reagente NitraVer5 (Hach) \\
Nitrato $\left(\mathrm{NO}{ }_{3}^{-}\right)$ & Espectrofotometria \\
Fósforo total $(\mathrm{P})$ & Fotometria de chama \\
Sódio $(\mathrm{Na})$ & Fotometria de chama \\
Potássio $(\mathrm{K})$ & Espectrofotometria por absorção atômica. \\
Cobre $(\mathrm{Cu})$ & Espectrofotometria por absorção atômica. \\
Zinco $(\mathrm{Zn})$ &
\end{tabular}

A fim de verificar se os valores efluentes dos cinco SAC(s) atendiam às pressuposições da casualidade, aplicaram-se os testes de Bartlett e Lillierfors, para verificar a homogeneidade e a normalidade, respectivamente, tomando-se as 11 coletas como repetições. Quando os dados apresentavam homogeneidade de variâncias e normalidade, procedeu-se a análise de variância, seguida do teste Tukey, a $5 \%$ de probabilidade, quando havia diferença significativa entre as médias. Nos casos em que os requisitos de homogeneidade ou de normalidade não foram atendidos, utilizou-se o teste não-paramétrico de Kruskal-Wallis, para comparar se havia ou não diferença significativa entre os dados (Ribeiro Júnior, 2001). 
MATOS, A. T.; FREITAS, W. S.; LO MONACO, P. A. V. Eficiência de sistemas alagados construídos na remoção de poluentes de águas residuárias da suinocultura. Ambi-Agua, Taubaté, v. 5, n. 2, p. 119-132, 2010. (doi:10.4136/ambi-agua.142)

\section{RESULTADOS E DISCUSSÃO}

Na Tabela 3 estão apresentadas as eficiências de remoção de sólidos totais (ST), sólidos suspensos totais (SST) e sólidos dissolvidos totais (SDT) e condutividade elétrica (CE) da ARS, no período de monitoramento dos 5 SAC(s).

Tabela 3. Eficiência na redução da condutividade elétrica (CE), sólidos totais (ST), sólidos suspensos totais (SST) e sólidos dissolvidos totais (SDT) obtida com base na análise de 11 amostras coletadas durante o período de monitoramento dos 5 SAC(s).

\begin{tabular}{|c|c|c|c|c|c|c|c|c|c|c|c|c|c|}
\hline \multirow[b]{2}{*}{ Variável } & \multirow[b]{2}{*}{ Tratamento } & \multicolumn{12}{|c|}{ Eficiência (\%)* } \\
\hline & & $\mathbf{1}^{\mathrm{a}}$ & $2^{a}$ & $3^{a}$ & $4^{\mathrm{a}}$ & $5^{a}$ & $6^{\mathrm{a}}$ & $7^{\mathrm{a}}$ & $\mathbf{8}^{\mathbf{a}}$ & $9^{a}$ & $10^{\mathrm{a}}$ & $11^{\mathrm{a}}$ & $\begin{array}{c}\text { Média } \pm \\
\text { (desvio-padrão) } \\
\end{array}$ \\
\hline \multirow{5}{*}{$\mathrm{CE}$} & SAC1 (Taboa) & 31 & 39 & 39 & 42 & 15 & 24 & 26 & 31 & 21 & 33 & 23 & $30 \pm 9$ \\
\hline & SAC2 (Alternanthera) & 64 & 52 & 52 & 29 & 13 & 21 & 32 & 48 & 22 & 53 & 20 & $37 \pm 17$ \\
\hline & SAC3 (Tifton 85) & 58 & 36 & 36 & 40 & 16 & 6 & 47 & 46 & 28 & 35 & 22 & $34 \pm 15$ \\
\hline & SAC4 (Misto) & 55 & 36 & 36 & 41 & 15 & 23 & 41 & 49 & 28 & 33 & 27 & $35 \pm 12$ \\
\hline & SAC 5 (Testemunha) & 65 & 38 & 38 & 37 & 23 & 34 & 25 & 28 & 25 & 37 & 19 & $33 \pm 13$ \\
\hline \multirow{5}{*}{ ST } & SAC1 (Taboa) & 19 & 48 & 73 & 43 & 63 & 72 & 72 & 43 & 35 & 58 & 36 & $51 \pm 18$ \\
\hline & SAC2 (Alternanthera) & 30 & 91 & 84 & 23 & 72 & 82 & 79 & 61 & 60 & 63 & 44 & $63 \pm 22$ \\
\hline & SAC3 (Tifton 85) & 29 & 96 & 94 & 51 & 75 & 82 & 86 & 52 & 62 & 66 & 49 & $67 \pm 21$ \\
\hline & SAC4 (Misto) & 26 & 89 & 78 & 56 & 79 & 83 & 81 & 68 & 54 & 56 & 61 & $66 \pm 18$ \\
\hline & SAC 5 (Testemunha) & 48 & 78 & 91 & 48 & 60 & 74 & 87 & 44 & 59 & 70 & 40 & $63 \pm 18$ \\
\hline \multirow{5}{*}{ SST } & SAC1 (Taboa) & 44 & 95 & 95 & 84 & 92 & 98 & 94 & 77 & 84 & 89 & 96 & $86 \pm 15$ \\
\hline & SAC2 (Alternanthera) & 83 & 97 & 98 & 68 & 99 & 100 & 98 & 94 & 94 & 94 & 96 & $93 \pm 9$ \\
\hline & SAC3 (Tifton 85) & 46 & 98 & 99 & 80 & 98 & 99 & 95 & 91 & 95 & 97 & 90 & $90 \pm 16$ \\
\hline & SAC4 (Misto) & 37 & 98 & 90 & 93 & 99 & 99 & 95 & 97 & 90 & 80 & 98 & $89 \pm 18$ \\
\hline & SAC 5 (Testemunha) & 95 & 94 & 96 & 91 & 97 & 98 & 98 & 92 & 94 & 93 & 96 & $95 \pm 2$ \\
\hline \multirow{5}{*}{ SDT } & SAC1 (Taboa) & 21 & 0 & 40 & 33 & 34 & 18 & 53 & 33 & -1 & 34 & -34 & $21 \pm 24$ \\
\hline & SAC2 (Alternanthera) & 18 & 81 & 49 & 13 & 19 & 44 & 57 & 38 & 31 & 23 & -15 & $33 \pm 25$ \\
\hline & SAC3 (Tifton 85) & 25 & 86 & 84 & 42 & 21 & 48 & 81 & 29 & 29 & 42 & 40 & $48 \pm 24$ \\
\hline & SAC4 (Misto) & 23 & 56 & 54 & 47 & 38 & 47 & 70 & 43 & 32 & 27 & 21 & $42 \pm 15$ \\
\hline & SAC 5 (Testemunha) & 56 & 70 & 80 & 38 & -29 & 37 & 73 & 9 & 31 & 51 & 25 & $40 \pm 32$ \\
\hline
\end{tabular}

*Baseada nos valores obtidos em amostras coletadas no afluente $(0 \mathrm{~m})$ e efluente $(24 \mathrm{~m})$.

Analisando-se os dados apresentados na Tabela 3, nota-se que as eficiências na remoção de ST apresentaram grandes oscilações, decorrentes da grande variação nas concentrações afluentes. Apesar de não haver diferença estatística $(p>0,05)$ entre as eficiências médias obtidas nos 5 SAC(s), o pior desempenho observado no SAC1, cultivado com taboa, pode estar associado à forma de propagação e desenvolvimento vegetativo da planta abaixo da superfície do substrato. Acredita-se que o desenvolvimento de rizomas possa ter aumentado a macroporosidade do substrato, uma vez que estes, por serem volumosos, podem ter suspendido a massa de brita em vários pontos do SAC. Além disso, o corte da taboa implicava morte de parte de caules e rizomas, ocorrendo decomposição destes, o que pode ter concorrido para a ocorrência da liberação de partículas sólidas na suspensão.

Os valores das eficiências médias de SST obtidos nos SAC(s) foram estatisticamente iguais entre si pelo teste de Kruskal-Wallis e pouco abaixo dos citados por Lee et al. (2004), que obtiveram eficiências de 96 a 99\% de SST no tratamento de ARS, em Taiwan. No entanto, em 45 das 55 medidas (82\% das medidas) as eficiências foram superiores a 90\%, o que pode estar associado à baixa velocidade de escoamento da água residuária e a grande área específica do meio suporte (US EPA, 2000). As eficiências obtidas neste trabalho estão próximas às obtidas por Brasil et al. (2007), que obtiveram eficiência média de $91 \%$, e superiores às obtidas por Valentim (2003) e Souza (2003), em cujos trabalhos foram obtidas remoções médias de SST de, respectivamente, 70 e 52\%, em SAC(s) cultivados com taboa, no 
tratamento de esgoto doméstico. Hench et al. (2003), no tratamento de esgoto doméstico em SAC(s), em pequena escala, obtiveram remoções de SST de 73 e 84\%, no $1^{\circ}$ ano, e de 83 e $79 \%$, no $2^{\circ}$ ano de operação do sistema, em SAC(s) cultivados (consórcio de taboa, Scirpus e junco) e não cultivados, respectivamente, não verificando também diferenças entre os SAC(s) e, tampouco, influência do tempo de operação do sistema.

Quanto aos sólidos dissolvidos totais (SDT), verificou-se que as eficiências na remoção destes variaram, em média, entre $21 \%$ e $48 \%$, não havendo diferença significativa entre estas $(p>0,05)$. Tais eficiências foram inferiores às obtidas por Souza (2003), em SAC(s) cultivados com taboa, que obteve eficiência média de 52\%. Embora as eficiências tenham sido estatisticamente iguais entre si, as maiores eficiências no SAC3 podem ser um indicativo de que a presença do capim tifton 85 seja importante para aumentar a remoção de nutrientes em solução na água residuária, o que pode ser decorrente da sua reconhecida capacidade em remover nutrientes do meio (Queiroz et al., 2004).

Na Tabela 4, estão apresentadas as eficiências de remoção dos atributos químicos, como demanda química de oxigênio (DQO), demanda bioquímica de oxigênio ( $\left.\mathrm{DBO}_{\text {total }}\right)$, nitrogênio total (N-total), íon amônio $\left(\mathrm{NH}_{4}^{+}\right)$, íon nitrato $\left(\mathrm{NO}_{3}{ }^{-}\right)$, fósforo (P-total), potássio $(\mathrm{K})$, sódio $(\mathrm{Na})$, cobre $(\mathrm{Cu})$ e zinco $(\mathrm{Zn})$, ao longo do período de monitoramento dos 5 SAC(s).

De acordo com os resultados apresentados na Tabela 4, verifica-se que as eficiências na remoção de DQO variaram entre 68 e 98\%, não havendo diferença significativa ( $>0,05)$ entre os valores médios, que variaram entre $87 \%$ e $92 \%$. Tais eficiências foram próximas às obtidas por Brasil et al. (2007) e superiores às obtidas por Valentim (2003), que obtiveram eficiências de $86 \%$ a $90 \%$ e $70 \%$, respectivamente. Lee et al. (2004), no tratamento de ARS, em Taiwan, encontraram remoções entre 77 e $84 \%$ na DQO, valores inferiores aos obtidos neste trabalho.

Em relação à $\mathrm{DBO}_{\text {total}}$, verificou-se que as eficiências obtidas em todas as amostras coletadas foram sempre superiores a $50 \%$, com médias, estatisticamente iguais entre si ( $>>0,05)$, variando entre 84 e 88\%. Esses resultados estão próximos aos encontrados por Roston (1994), que obteve, no tratamento de esgoto nos EUA, em SAC cultivado com taboa, reduções de 165 para $13 \mathrm{mg} \mathrm{L}^{-1}$ na concentração de $\mathrm{DBO}_{\text {total }}$ (eficiência de 92\%), e superiores aos obtidos em SAC não cultivado, onde foi obtida redução de 165 para $62 \mathrm{mg} \mathrm{L}^{-1}$ (eficiência de 62\%). Já Gersberg et al. (1985, apud Reed, 1995), em SAC(s) pilotos para tratamento de esgoto doméstico, com concentração afluente de $118 \mathrm{mg} \mathrm{L}^{-1}$, encontraram nos SAC(s) cultivados com Phragmites, Scirpus, Typha e não cultivado, efluentes iguais a 5, 22, 30 e 36 $\mathrm{mg} \mathrm{L}^{-1}$, respectivamente, concluindo haver, diferentemente dos resultados obtidos neste trabalho, maiores eficiências em SAC(s) cultivados e naqueles que tinham maior profundidade radicular.

No que se refere ao atendimento aos padrões de lançamento de efluentes estabelecidos na legislação vigente no Estado de Minas Gerais, Deliberação Normativa COPAM/CERH N ${ }^{0}$ 01/2008 de que o sistema de tratamento deva apresentar eficiência mínima de $70 \%$ na remoção de DQO e $75 \%$ na remoção da $\mathrm{DBO}_{\text {total, }}$ os resultados apresentados na Tabela 3 só não foram atendidos uma única vez para DQO e sete vezes para $\mathrm{DBO}_{\text {total. }}$. No que se refere aos valores médios de DQO, o efluente apresentou condições de lançamento em corpos hídricos em todos os SAC(s), enquanto que apenas no SAC2, ainda que se encontrassem muito próximos dos $85 \%$ exigidos, o valor de $\mathrm{DBO}_{\text {total }}$ não seria atendido.

Maiores eficiências de remoção da DQO, quando comparadas à de $\mathrm{DBO}_{\text {total, }}$, se devem ao fato de que remoções consideráveis da carga orgânica nos SAC(s) se deram, principalmente, por mecanismos físicos. Tal constatação pode ser confirmada na Figura 2 em que a tendência de redução dos valores da relação $\mathrm{DQO} / \mathrm{DBO}_{\text {total }}$ ao longo dos SAC(s) indica que estes foram mais eficientes na remoção de material inerte (não biodegradável). Além disso, segundo Von Sperling (2005), por terem os afluentes valores da relação $\mathrm{DQO} / \mathrm{DBO}_{\text {total }}$ superiores a 3,5, a fração inerte da ARS pode ser considerada elevada. 
MATOS, A. T.; FREITAS, W. S.; LO MONACO, P. A. V. Eficiência de sistemas alagados construídos na remoção de poluentes de águas residuárias da suinocultura. Ambi-Agua, Taubaté, v. 5, n. 2, p. 119-132, 2010. (doi:10.4136/ambi-agua.142)

Tabela 4. Eficiência na remoção de DQO, $\mathrm{DBO}_{\text {total, }} \mathrm{N}$-total, $\mathrm{N}-\mathrm{NH}_{4}{ }^{+}, \mathrm{N}-\mathrm{NO}_{3}{ }^{-}, \mathrm{P}, \mathrm{K} \mathrm{Na}$, Cu e Zn, obtida com base na análise de 11 amostras coletadas ao longo do período de monitoramento dos 5 SAC(s).

\begin{tabular}{|c|c|c|c|c|c|c|c|c|c|c|c|c|c|}
\hline \multirow{2}{*}{ Variável } & \multirow[b]{2}{*}{ Tratamento } & \multicolumn{12}{|c|}{ Eficiência (\%)* } \\
\hline & & $1^{\mathrm{a}}$ & $2^{\mathrm{a}}$ & $3^{\mathrm{a}}$ & $4^{\mathrm{a}}$ & $5^{\mathrm{a}}$ & $6^{\mathrm{a}}$ & $7^{\mathrm{a}}$ & $8^{\mathbf{a}}$ & $9^{a}$ & $10^{\mathrm{a}}$ & $11^{\mathrm{a}}$ & $\begin{array}{c}\text { Média } \pm \\
(d p)^{*}\end{array}$ \\
\hline \multirow{5}{*}{ DQO } & SAC1 (Taboa) & 76 & 90 & 95 & 71 & 94 & 96 & 96 & 79 & 87 & 90 & 89 & $88 \pm 9$ \\
\hline & SAC2 (Alternanthera) & 93 & 91 & 97 & 68 & 91 & 97 & 98 & 80 & 85 & 74 & 87 & $87 \pm 10$ \\
\hline & SAC3 (Tifton 85) & 90 & 92 & 98 & 70 & 94 & 98 & 98 & 79 & 89 & 93 & 88 & $90 \pm 9$ \\
\hline & SAC4 (Misto) & 97 & 86 & 93 & 87 & 97 & 98 & 97 & 89 & 89 & 92 & 91 & $92 \pm 4$ \\
\hline & SAC5 (Testemunha) & 94 & 78 & 98 & 73 & 93 & 95 & 99 & 83 & 88 & 93 & 91 & $89 \pm 8$ \\
\hline \multirow{5}{*}{$\mathrm{DBO}_{\text {total }}$} & SAC1 (Taboa) & & 92 & 86 & 50 & 89 & 96 & 91 & 83 & 89 & 93 & 89 & $86 \pm 13$ \\
\hline & SAC2 (Alternanthera) & & 92 & 87 & 66 & 85 & 95 & 93 & 80 & 92 & 64 & 89 & $84 \pm 11$ \\
\hline & SAC3 (Tifton 85) & & 86 & 96 & 63 & 91 & 97 & 94 & 84 & 91 & 92 & 88 & $88 \pm 10$ \\
\hline & SAC4 (Misto) & & 90 & 96 & 66 & 94 & 98 & 98 & 84 & 87 & 92 & 74 & $88 \pm 11$ \\
\hline & SAC 5 (Testemunha) & & 84 & 94 & 66 & 85 & 95 & 95 & 80 & 89 & 91 & 81 & $86 \pm 9$ \\
\hline \multirow{5}{*}{ N-Total } & SAC1 (Taboa) & 57 & 69 & 60 & 54 & 41 & 54 & 36 & 42 & 41 & 61 & 41 & $51 \pm 11$ \\
\hline & SAC2 (Alternanthera) & 83 & 87 & 73 & 51 & 48 & 57 & 54 & 60 & 46 & 71 & 36 & $61 \pm 16$ \\
\hline & SAC3 (Tifton 85) & 73 & 92 & 86 & 53 & 59 & 67 & 63 & 66 & 53 & 55 & 35 & $64 \pm 16$ \\
\hline & SAC4 (Misto) & 79 & 81 & 65 & 61 & 61 & 66 & 60 & 66 & 51 & 62 & 54 & $64 \pm 9$ \\
\hline & SAC 5 (Testemunha) & 79 & 74 & 73 & 55 & 52 & 53 & 58 & 41 & 41 & 63 & 32 & $57 \pm 15$ \\
\hline \multirow{5}{*}{$\mathrm{N}-\mathrm{NH}_{4}^{+}$} & SAC1 (Taboa) & 90 & 62 & 21 & 60 & 23 & 32 & 27 & 35 & 59 & 44 & 55 & $46 \pm 21$ \\
\hline & SAC2 (Alternanthera) & 98 & 75 & 46 & 61 & 16 & 32 & 26 & 66 & 42 & 66 & 39 & $52 \pm 24$ \\
\hline & SAC3 (Tifton 85) & 97 & 93 & 18 & 67 & 11 & 58 & 64 & 61 & 57 & 39 & 45 & $56 \pm 27$ \\
\hline & SAC4 (Misto) & 96 & 69 & 12 & 73 & 37 & 44 & 52 & 67 & 36 & 43 & 50 & $53 \pm 23$ \\
\hline & SAC 5 (Testemunha) & 98 & 76 & 29 & 65 & 35 & 45 & 37 & 38 & 61 & 64 & 43 & $54 \pm 21$ \\
\hline \multirow{5}{*}{$\mathrm{N}-\mathrm{NO}_{3}{ }^{-}$} & SAC1 (Taboa) & -381 & 32 & -55 & 6 & 96 & -4485 & -271 & -184 & -109 & 14 & -94 & -494 \\
\hline & SAC2 (Alternanthera) & -52 & 58 & -2868 & 9 & 97 & -892 & -135 & -108 & -63 & 13 & -66 & -364 \\
\hline & SAC3 (Tifton 85) & 52 & 82 & -398 & -110 & -375 & -47100 & -1717 & -948 & -16 & -20 & -600 & -4650 \\
\hline & SAC4 (Misto) & -3 & 0 & 19 & 7 & 44 & -143 & -84 & -72 & -127 & 7 & -70 & -38 \\
\hline & SAC5 (Testemunha) & -51 & 64 & -67 & 22 & 79 & -304 & -475 & -214 & -90 & 16 & -258 & -116 \\
\hline \multirow{5}{*}{$\mathrm{P}$} & SAC1 (Taboa) & 10 & 41 & 56 & 35 & 42 & 57 & 38 & 25 & 15 & 11 & 33 & $33 \pm 16 \mathrm{~A}$ \\
\hline & SAC2 (Alternanthera) & 54 & 77 & 72 & 57 & 49 & 70 & 62 & 51 & 40 & 34 & 37 & $55 \pm 14$ B \\
\hline & SAC3 (Tifton 85) & 7 & 71 & 87 & 50 & 53 & 80 & 58 & 43 & 62 & 51 & 47 & $55 \pm 21$ B \\
\hline & SAC4 (Misto) & 56 & 78 & 62 & 33 & 63 & 69 & 58 & 52 & 28 & 38 & 50 & $\mathrm{AB}$ \\
\hline & SAC 5 (Testemunha) & 61 & 69 & 80 & 39 & 39 & 74 & 75 & 35 & 58 & 42 & 37 & $55 \pm 17 \mathrm{~B}$ \\
\hline \multirow{5}{*}{$\mathrm{K}$} & SAC1 (Taboa) & 42 & 73 & 59 & 42 & 17 & 13 & 28 & 8 & 11 & 19 & 6 & $29 \pm 22$ \\
\hline & SAC2 (Alternanthera) & 75 & 85 & 70 & 25 & 13 & 31 & 48 & 54 & 26 & 66 & 17 & $46 \pm 25$ \\
\hline & SAC3 (Tifton 85) & 21 & 87 & 53 & 30 & 16 & 29 & 53 & 37 & 6 & 33 & 6 & $34 \pm 24$ \\
\hline & SAC4 (Misto) & 44 & 40 & 48 & 25 & 20 & 23 & 53 & 50 & 37 & 19 & 21 & $34 \pm 13$ \\
\hline & SAC 5 (Testemunha) & 67 & 71 & 48 & 32 & 9 & 15 & 37 & 27 & 23 & 28 & 12 & $34 \pm 21$ \\
\hline \multirow{5}{*}{$\mathrm{Na}$} & SAC1 (Taboa) & 33 & 48 & 41 & 26 & -4 & 2 & 8 & 0 & 19 & 30 & 0 & $18 \pm 18$ \\
\hline & SAC2 (Alternanthera) & 69 & 29 & 57 & 5 & 7 & 2 & 22 & 28 & -4 & 60 & -2 & $25 \pm 27$ \\
\hline & SAC3 (Tifton 85) & -23 & 63 & 35 & 11 & -7 & 17 & 30 & 25 & 4 & 33 & 6 & $18 \pm 23$ \\
\hline & SAC4 (Misto) & 13 & 65 & 39 & 27 & 9 & 15 & 10 & 41 & 14 & 28 & 9 & $24 \pm 18$ \\
\hline & SAC 5 (Testemunha) & 44 & 65 & 30 & 26 & 9 & 17 & 30 & 16 & 24 & 43 & 2 & $28 \pm 18$ \\
\hline \multirow{5}{*}{$\mathrm{Cu}$} & SAC1 (Taboa) & 17 & 92 & 100 & 99 & 100 & 27 & 100 & 100 & - & 100 & 73 & $81 \pm 32$ \\
\hline & SAC2 (Alternanthera) & 17 & 93 & 100 & -17 & 100 & 100 & -18 & 79 & 18 & 100 & 99 & $61 \pm 50$ \\
\hline & SAC3 (Tifton 85) & - & 94 & 100 & - & 98 & 100 & 85 & 100 & 50 & 100 & 87 & $90 \pm 16$ \\
\hline & SAC4 (Misto) & -3 & -538 & 100 & 33 & 100 & 100 & 58 & 100 & 60 & 100 & 94 & $19 \pm 188$ \\
\hline & SAC 5 (Testemunha) & -121 & -20 & 100 & 44 & 100 & -386 & 100 & 100 & 72 & 100 & 100 & $17 \pm 151$ \\
\hline \multirow{5}{*}{$\mathrm{Zn}$} & SAC1 (Taboa) & 47 & 100 & 100 & - & 100 & 99 & 100 & 95 & 100 & 98 & 95 & $93 \pm 16$ \\
\hline & SAC2 (Alternanthera) & 100 & 100 & 100 & 100 & 100 & 100 & 100 & 98 & 52 & 97 & 100 & $95 \pm 14$ \\
\hline & SAC3 (Tifton 85) & 24 & 100 & 100 & - & 100 & 99 & 100 & 100 & 59 & 98 & 100 & $88 \pm 26$ \\
\hline & SAC4 (Misto) & 100 & 98 & 100 & 100 & 100 & 100 & 96 & 100 & 85 & 98 & 100 & $98 \pm 5$ \\
\hline & SAC 5 (Testemunha) & 100 & 100 & 100 & - & 100 & 99 & 100 & 100 & 82 & 96 & 95 & $97 \pm 6$ \\
\hline
\end{tabular}

*Baseada nos valores obtidos em amostras coletadas no afluente $(0 \mathrm{~m})$ e efluente $(24 \mathrm{~m})$. 
As eficiências na remoção de N-total foram estatisticamente iguais entre os SAC(s) $(\mathrm{p}>0,05)$ e superiores às encontradas por Hussar (2001), em SAC(s) cultivados com taboa $\mathrm{DBO}_{\text {total }}$ para tratamento de ARS, que encontrou eficiências variando entre 9 e $71 \%$ e média de 35\%. Comparando-se com trabalhos que utilizaram esgoto doméstico, cujos níveis de Ntotal são inferiores aos valores encontrados na ARS, tais eficiências foram semelhantes às obtidas por Souza e Bernardes (1996, apud Valentim,2003), Mansor (1998), Valentim (1999) e Brasil et al. (2007), que obtiveram eficiências de 53\%, 55\%, 48\% e 57\% (tempo de detenção hidráulica de 3,8 dias), respectivamente; e superiores às obtidas por Tunçsiper et al. (2004), Brix (1994), Börner (1992, apud Kuschk et al., 2003) e Brasil et al. (2007), que obtiveram eficiências de 45\% (tempo de detenção hidráulica de 0,75 a 2,93 dias), 39,6\%, 40\% (em 268 SAC(s) avaliados na Europa) e 33\% (tempo de detenção hidráulica de 2 dias).

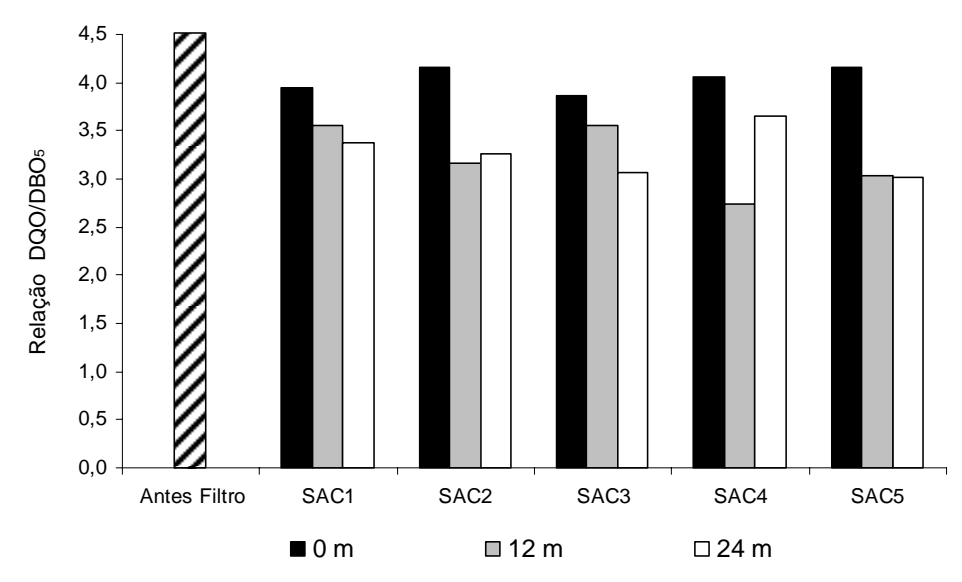

Figura 2. Valores médios da variação $\mathrm{DQO} / \mathrm{DBO}_{\text {total }}$ nos pontos antes do filtro, a 0, 12 e 24 metros dos cinco SAC(s).

Hench et al. (2003), no tratamento de esgoto doméstico em SAC(s) em pequena escala, obtiveram remoções de 74 e $29 \%$, no $1^{\circ}$ ano, e de 31 e $5 \%$, no $2^{\circ}$ ano de monitoramento, no N-total, em SAC(s) cultivados (consórcio de taboa, Scirpus e junco) e não cultivados, respectivamente, concluindo ocorrerem maiores eficiências em SAC(s) cultivados. Além disso, esses autores concluíram haver redução significativa nas eficiências de remoção de Ntotal com o tempo de operação do sistema.

As eficiências médias na remoção de amônio dos cinco SAC(s) foram estatisticamente iguais entre si (p>0,05) e superiores à média de 30\%, obtida em 268 SAC(s) avaliados na Europa por Börner (1992, apud Kuschk et al., 2003) e também às obtidas por Stone et al. (2004), que encontrou eficiência média de 33\% tratando ARS em SAC(s) cultivados com misto de Scirpus e taboa nos EUA.

Embora tenha havido muita oscilação nos valores obtidos, a concentração média efluente de nitrato, quando comparadas ao afluente médio de todos os SAC(s), aumentou, já que passou de $1,5 \mathrm{mg} \mathrm{L}^{-1}$ para $2,5 \mathrm{mg} \mathrm{L}^{-1}$, proporcionando eficiências médias negativas, que variaram de -38 a $-4.650 \%$, porém, estatisticamente iguais entre si $(p>0,05)$ pelo teste de Kruskall-Walliss. $\mathrm{O}$ aumento na concentração de nitrato pode ter ocorrido devido à oxidação de formas amoniacais, o que possivelmente proporcionou concentrações efluentes superiores às efluentes.

De acordo com os dados apresentados na Tabela 4, a eficiência média de remoção de Ptotal nos SAC2, SAC3, SAC4 e SAC5 foram estatisticamente iguais entre si $(\mathrm{p}>0,05)$, com valores superiores aos obtidos por Valentim (2003), Tunçsiper et al. (2004) e Brasil et al. (2007), que obtiveram eficiência média de remoção de P-total de 23\% (TDH entre 2 e 4 dias), $38 \%$ (TDH de 0,75 a 2,9 dias) e de 31 a $48 \%$ (TDH de 1,9 a 3,8 dias), respectivamente, em SAC(s) cultivados com taboa para o tratamento de esgoto doméstico. Tobias (2002), no Brasil, e Stone et al. (2004), nos EUA, trabalhando em SAC(s) cultivados com taboa para tratamento de ARS, encontraram eficiências muito baixas para $\mathrm{P}$, sendo que o primeiro 
encontrou, em algumas amostras, aumento na concentração efluente em relação à afluente. O fato de não terem sido encontradas diferenças significativas entre SAC(s) plantados e não plantado sugere que grande parte do P-total foi removido por acúmulo de fósforo orgânico no meio-suporte e devido à sua imobilização pelos micro-organismos, tal como observado por Lee et al. (2004), ao utilizarem SAC(s) no tratamento de ARS, em Taiwan, que concluíram que $97,3 \%$ da remoção do P-total ocorreu por mecanismos puramente físicos, 2,1\% por mecanismos microbiológicos e, apenas, $0,3 \%$ por extração pelas plantas (Eichhornia crassipes). Entretanto, torna-se importante lembrar que a maior perda de água, por evapotranspiração, nos SAC(s) cultivados causou concentração de solutos, o que pode ter subestimado a maior eficiência obtida em sistemas cultivados.

Não existe muita informação sobre o comportamento de metais alcalinos em SAC(s) utilizados no tratamento de águas residuárias, uma vez que esse elemento químico não está, normalmente, associado à qualidade do efluente de sistema de tratamento. Todavia, o potássio e o sódio estão incluídos entre os nutrientes absorvidos pelos vegetais e que podem causar problemas quando presentes em altas concentrações no solo (Matos e Sediyama, 1995), prejudicando um possível aproveitamento agrícola do efluente dos SAC(s). Por essa razão, suas concentrações devem ser, necessariamente, mensuradas. Analisando os resultados apresentados na Tabela 4, nota-se grande variação nas eficiências de remoção de $K$ ao longo do período de tempo de coletas de amostras do afluente e efluente dos SAC(s), entretanto, não houve diferença significativa ( $>00,05$ ) entre os valores médios, que variaram de $29 \%$, no SAC1, a 46\%, no SAC2, cultivado com Alternanthera, maior extratora de $\mathrm{K}$ dentre as espécies vegetais avaliadas. Esses resultados de remoção estão próximos aos reportados por Brasil et al. (2007), que relataram terem obtido, em SAC(s) cultivados com taboa, no tratamento de esgoto doméstico, valores de eficiência média de 35\% (tempo de detenção hidráulica de 1,9 dias) e 52\% (tempo de detenção hidráulica de 3,9 dias).

Não foi observada diferença significativa $(\mathrm{p}>0,05)$ entre as médias das eficiências nos cinco SAC(s). O fato de não se ter obtido maiores remoções em SAC(s) cultivados é justificado pela maior concentração de nutrientes em seus efluentes, decorrente da maior evapotranspiração de água, ocorrente nos SAC(s) cultivados, quando comparados ao SAC5, que não ficou submetido ao efeito conjunto da transpiração pelas plantas com a evaporação direta nos leitos. A alta evapotranspiração em SACs foi reportada por Brasil e Matos (2008), que obtiveram evapotranspiração média de $9,3 \mathrm{~mm} \mathrm{~d}^{-1}$, resultando num coeficiente da cultura (Kc) da taboa com variação de 2,22 a 4,58.

Entre os nutrientes avaliados neste trabalho, o $\mathrm{Na}^{+}$foi o que apresentou menores eficiências médias de remoção, tendo sido obtidos valores entre 28\% (SAC5) e 18\% (SAC1). Ainda assim, tais remoções foram superiores às obtidas por Brasil et al. (2007), que relatou ter obtido, em SAC(s) cultivados com taboa, no tratamento de esgoto doméstico, valores de eficiência média de 5\% (TDH de 1,9 dias) e 7\% (tempo de detenção hidráulica de 3,9 dias) na remoção de $\mathrm{Na}^{+}$, justificando o insucesso da remoção de $\mathrm{Na}^{+}$à sua relativamente alta concentração na água residuária (entre 25 e $50 \mathrm{mg} \mathrm{L}^{-1}$ ), à grande solubilidade, à baixa absorção pelas plantas e à pequena associação do cátion com o material orgânico.

Não foi observada diferença significativa $(\mathrm{p}>0,05)$, pelo teste de Kruskal-Wallis, entre os valores médios de eficiência de remoção de $\mathrm{Cu}$, no entanto, analisando-se os dados, nota-se grande variação nas eficiências de remoção de $\mathrm{Cu}$ entre os períodos de coletas de amostras do afluente e efluente e entre os SAC(s), sendo que em algumas coletas houve, inclusive, aumento na concentração efluente em relação à afluente, ocasionando valores negativos de eficiência. Em outras ocasiões, a concentração afluente foi igual a zero, já que os filtros orgânicos são reconhecidamente (Matos et al., 2003; Lo Monaco et al., 2004) eficientes na remoção de metais pesados, não possibilitando o cálculo da eficiência de remoção do metal. Observou-se, ainda, que parte dos valores de remoção de $\mathrm{Cu}$, estão superiores (SAC1 e SAC3) e parte estão inferiores (SAC4 e SAC 5) aos 52-62\% e 59-67\%, encontrados por Lim et al. (2001), em SAC(s) cultivados (taboa) e não cultivados, respectivamente, e aos valores 
de eficiência entre 33 e 66 \% (estimados), encontrados por Tobias (2002), no tratamento de ARS em SAC(s) de $3 \mathrm{~m}$ x 1,4 m, cultivados com taboa.

As eficiências de remoção de Zn variaram entre 24 e 100\%, em 46 das 52 amostragens válidas, esses valores foram superiores a 95\%, demonstrando ser o sistema bastante eficiente na remoção de Zn. Esses resultados de eficiência estão superiores aos valores médios obtidos por Tobias (2002), no tratamento de efluentes de ARS de reatores anaeróbios em SAC(s) de 3 m x 1,4 m, cultivados com taboa, que encontrou eficiências entre 40 e $80 \%$ (estimado). Atribui-se à sedimentação, junto com a biomassa produzida, e à absorção pelas plantas, a remoção do Zn da água residuária.

\section{CONCLUSÕES}

Pôde-se concluir que os cinco SAC(s) tiveram eficiências de remoção de poluentes estatisticamente iguais entre si, sendo que tais sistemas foram mais eficientes na remoção de SST, DQO, $\mathrm{DBO}_{\text {total }}$ e Zn, com valores médios, considerando-se os cinco SAC(s) de 91, 89, 86 e 94\%, respectivamente. No entanto, foram obtidas remoções consideráveis também nas concentrações de ST, N-total, $\mathrm{NH}_{4}{ }^{+}$e P-total com valores médios de 62, 59, 52 e 50\%, respectivamente.

As plantas se equivaleram entre si, em termos de conferir eficiência ao sistema. O SAC não cultivado apresentou capacidade semelhante na remoção de poluentes da ARS que os SAC(s) cultivados, entretanto, ainda que não tenham sido obtidas diferenças significativas, há de se considerar que tenha sido obtida tendência de maior remoção de macro e micronutrientes nos SACs cultivados com capim tifton, alternathera e sistema misto.

Ainda que não tenha sido objeto de avaliação, verificou-se menor entupimento dos poros do meio nos SACs cultivados que no não cultivado, o que pode ser considerado aspecto de muita importância operacional dos SACs.

\section{REFERÊNCIAS BIBLIOGRÁFICAS}

AMERICAN PUBLIC HEALTH ASSOCIATION - APHA. Standard methods for the examination of water and wastewater. 21. ed. Washington: APHA, 2005. 1600p.

ARIAS, C. A.; BRIX, H. Phosphorus removal in constructed wetlands: Can suitable alternative media be identified? In: INTERNATIONAL CONFERENCE ON WASTE STABILISATION PONDS AND 9TH INTERNATIONAL CONFERENCE ON WETLAND SYSTEMS FOR WATER POLLUTION CONTROL, 6., Sept./Out. 2004, Avignon. Proceedings... Avignon: IWA, 2004. 1 CD-ROM.

BRASIL, M. S.; MATOS, A. T.; SILVA, C. M.; CECON, P. R.; SOARES, A. A. Modeling of pollution removal in constructed wetlands with horizontal subsurface flow. Agricultural Engineering Research, v. 13, n. 2, p. 48-56, 2007.

BRASIL, M. S.; MATOS, A. T. Avaliação de aspectos hidráulicos e hidrológicos de sistemas alagados construídos de fluxo subsuperficial. Eng. Sanitária e Ambiental, v. 13, n. 3, p. 323-328, 2008.

BRIX, H. Functions of macrophytes in constructed wetlands. Water Science Technology, v. 29, n. 4, p. 71-78, 1994.

HENCH, K.; BISSONNETTE, G. K; SEXSTONE, A. J; COLEMAN, J. G; GARBUTT, K.; KOUSEN, J. G. Fate of physical, chemical, and microbial contaminants in domestic wastewater follwing treatment by small constructed wetlands. Water Research, v. 37, n. 4, p. 921-927, 2003. 
MATOS, A. T.; FREITAS, W. S.; LO MONACO, P. A. V. Eficiência de sistemas alagados construídos na remoção de poluentes de águas residuárias da suinocultura. Ambi-Agua, Taubaté, v. 5, n. 2, p. 119-132, 2010. (doi:10.4136/ambi-agua.142)

HUSSAR, G. J. Avaliação do desempenho de leitos cultivados no tratamento de águas residuárias de suinocultura. 2001. 118f. Dissertação (Mestrado em Engenharia Agrícola) - FEAGRI-UNICAMP, Campinas, 2001.

JUWARKAR, A. S. Domestic treatment through constructed wetland in India. Water Science and Technology, v. 32, n. 3, p. 291-294, 1995.

KUSCHK, P.; WIEBNER, A.; KAPPELMEYER, U.; WEIBBRODT, E.; KÂSTNER, M.; STOTTMEISTER, U. Annual cycle of nitrogen removal by a pilot-scale subsurface horizontal flow in a constructed wetland under moderate climate. Water Research, v. 37, p. 4236-4242, 2003.

LAUTENSCHLAGER, S. R. Modelagem do desempenho de Wetlands construídas. 2001. 90f. Dissertação (Mestrado em Engenharia Hidráulica e Sanitária) - Escola Politécnica da Universidade de São Paulo, São Paulo, 2001.

LEE, C. Y.; LEE, C. C.; LEE, F. Y.; TSENG, S. K.; LIAO, C. J. Performance of subsurface flow constructed wetland taking pretead swine effluet under heavy loads. Bioresource Technology, n. 92, p. 173-179, 2004.

LIM, P. E.; WONG, T. F.; LIM, D. V. Oxygen demand, nitrogen and copper removal by freewater-surface and subsurface-flow constructed wetlands under tropical conditions. Environment International, v. 26, n. 5-6, p. 425-431, 2001.

LO MONACO, P. A.; MATOS, A. T.; JORDÃO, C. P.; CECON, P. C.; MARTINEZ, M. A. Influência da granulometria da serragem de madeira como material filtrante no tratamento de águas residuárias. Revista Brasileira de Engenharia Agrícola e Ambiental, Campina Grande, v. 8, n. 1, p. 116-119, 2004.

MAGALHÃES, M. A.; MATOS, A. T.; DENICULI, W.; TINOCO, I. F. F. Operação de filtros orgânicos utilizados no tratamento de águas residuárias de suinocultura. Revista Brasileira de Engenharia Agrícola e Ambiental, Campina Grande, v. 10, n. 2, p. $472-$ 478, 2006.

MANDER, U.; LÕHMUS, K.; KUUSEMETS, V.; TEITER, S.; NURK, K. Dynamics of nitrogen and phosphorus budgets in a horizontal subsurface flow constructed wetland. In: INTERNATIONAL CONFERENCE ON WASTE STABILISATION PONDS AND 9TH INTERNATIONAL CONFERENCE ON WETLAND SYSTEMS FOR WATER POLlution CONTROL, 6., Sept./Out. 2004, Avignon. Proceedings... Avignon: IWA, 2004. 1 CD-ROM.

MANSOR, M. T. C. Uso de leito de macrófitas no tratamento de águas residuárias. 1998. 106f. Dissertação (Mestrado em Engenharia Agrícola) - Universidade Estadual de Campinas, Campinas, 1998.

MARQUES, D. M. Terras úmidas construídas de fluxo subsuperficial. In: CAMPOS, R. (Coord.). Tratamento de esgotos sanitários por processo anaeróbio e disposição controlada no solo. Rio de Janeiro: ABES, 1999. p. 409-435.

MATOS, A. T.; BRANDÃO, V. S.; NEVES, J. C. L.; MARTINEZ, M. A. Removal of Cu and $\mathrm{Zn}$ from swine raising wastewater using organic filters. Environmental Technology, v. 24, n. 2, p. 171-178, 2003.

MATOS, A. T.; SEDIYAMA, M. A. N. Riscos potenciais ao ambiente pela aplicação de dejetos líquido de suínos ou compostos orgânicos no solo. In: SEMINÁRIO MINEIRO SOBRE MANEJO E UTILIZAÇÃO DE DEJETOS DE SUÍNOS, 1., 1995, Ponte Nova. Anais... Ponte Nova: EPAMIG/EMATER/UFV/ASSUVAP, 1995. p. 45-54. 
NELSON, E. A.; SPECHT, W. A. L.; BOWERS, J. A.; GLADDEN, J. B. Constructed wetlands for removal of heavy metals from nudes outfall effluent. Aiken: Westinghouse Savannah River Company, 2002. Disponível em: <http://sti.srs.gov/fulltext/ms2002600/ms2002600.html> Acesso em: 16 set. 2004.

QUEIROZ, F. M.; MATOS, A. T.; PEREIRA, O. G.; OLIVEIRA, R. A.; LEMOS, A. L. Características químicas do solo e absorção de nutrientes por gramíneas em rampas de tratamento de águas residuárias da suinocultura. Revista Engenharia na Agricultura, v. 012, n. 2, 2004, p. 77-90.

REED, S, C.; CRITES, R. W.; MIDDLEBROOKS, E. J. Natural systems for management and treatment. New York: McGraw-Hill, 1995. 435p.

RIBEIRO JÚNIOR, J. I. Análises estatísticas no SAEG. Viçosa, MG: UFV, 2001. 301p.

ROSTON, D. M. Uso de várzeas artificiais para tratamento de efluente de tanque séptico. In: CONGRESSO BRASILEIRO DE ENGENHARIA AGRÍCOLA, 23., 1994, Campinas. Anais ... Campinas: ABEA, 1994. p. 210.

SHUTES, R. B. E. Artificial wetlands and water quality improvement. Environment International, v. 26, n. 5/6, p. 441-447, 2001.

SOUSA, J. T.; Van HAANDEL, A. C.; COSENTINO, P. R. S.; GUIMARÃES, A. V. A. Póstratamento de efluente de reator UASB utilizando sistemas "wetlands" construídos. Revista Brasileira de Engenharia Agrícola e Ambiental, v. 4, n. 1, p. 87-91, 2000.

SOUSA, J. T.; Van HAANDEL, A. C.; GUIMARÃES, A. V. A. Acumulação de fósforo em sistemas wetlands. In: CONGRESSO BRASILEIRO DE ENGENHARIA SANITÁRIA E AMBIENTAL, 21., 2001, João Pessoa. Anais... João Pessoa: FITABES, 2001. 1 CDROM.

SOUZA A. L. Estudo experimental e numérico do processo de remoção de poluentes nos leitos cultivados. 2003. 141f. Dissertação (Mestrado em Engenharia Agrícola) Universidade Estadual de Campinas, Campinas, 2003.

STONE, K. C.; POACH, M. E.; HUNT, P. G.; REEDY, G. B.; Marsh-pond-marsh constructed wetland design analysis for swine lagoon wastewater treatment. Ecological Engineering, n. 23, p.127-133, 2004.

TOBIAS, A. C. T. Tratamento de resíduos da suinocultura: uso de reatores anaeróbios sequenciais seguido de leitos cultivados. 2002. 123f. Dissertação (Doutorado em Engenharia Agrícola) - Universidade Estadual de Campinas, Campinas, 2002.

TUNÇSIPER, B.; AYAZ, S. Ç.; AKÇA, L. Performances analysis and modeling of an experimental constructed wetlands. In: INTERNATIONAL CONFERENCE ON WASTE STABILISATION PONDS AND 9TH INTERNATIONAL CONFERENCE ON WETLAND SYSTEMS FOR WATER POLLUTION CONTROL, 6., Sept./Out. 2004, Avignon. Proceedings... Avignon: IWA, 2004. 1 CD-ROM.

UNITED STATES ENVIRONMENTAL PROTECTION AGENCY - EPA. Manual constructed wetlands for municipal wastewater treatment. Cicinnati: EPA, 2000. Disponível em: <http://www.epa.gov/ORD/NRMRL>. Acesso: 15 fev. 2005.

VALENTIM, M. A. A. Uso de leitos cultivados no pós-tratamento de tanque séptico modificado. 1999. 119f. Dissertação (Mestrado em Engenharia Agrícola) - Faculdade de Engenharia Agrícola, Universidade Estadual de Campinas, Campinas, 1999. 
MATOS, A. T.; FREITAS, W. S.; LO MONACO, P. A. V. Eficiência de sistemas alagados construídos na remoção de poluentes de águas residuárias da suinocultura. Ambi-Agua, Taubaté, v. 5, n. 2, p. 119-132, 2010. (doi:10.4136/ambi-agua.142)

VALENTIM, M. A. A. Desempenho de leitos cultivados (“construted wetland”) para tratamento de esgoto: contribuições para concepção e operação. 2003. 210f. Tese (Doutorado em Engenharia Agrícola) - Faculdade de Engenharia Agrícola, Universidade Estadual de Campinas, Campinas, 2003.

VON SPERLING. M. Introdução à qualidade das águas e ao tratamento de esgotos. 3. ed. Belo Horizonte: DESA/UFMG, 2005. 452p.

VYMAZAL, J. Removal of phosphorus via harvesting of emergent vegetation in constructed wetlands for wastewater treatment. In: INTERNATIONAL CONFERENCE ON WASTE STABILISATION PONDS AND 9TH INTERNATIONAL CONFERENCE ON WETLAND SYSTEMS FOR WATER POLLUTION CONTROL, 6., Sept./Out. 2004, Avignon. Proceedings... Avignon: IWA, 2004. 1 CD-ROM.

WOOD, A. Constructed wetlands in water pollution control: fundamentals to their understanding. Water Science and Tecnology, v. 32, n. 3, p. 21-29, 1995. 